\title{
Voids in the Local Volume: a Limit on Appearance of a Galaxy in a Dark Matter Halo
}

\author{
Anton V. Tikhonov ${ }^{1}$ and Anatoly A. Klypin ${ }^{2} \dagger$ \\ ${ }^{1}$ Chair of Astrophysics, Department of Mathematics and Mechanics, St.Petersburg \\ State University, Universitetsky prospect, 28, Saint-Petersburg, Petrodvoretz, \\ 198504 Russian Federation \\ email: ti@hotbox.ru, avt@gtn.ru \\ ${ }^{2}$ The Astronomy Department, New Mexico State University, \\ Las Cruces, New Mexico 88003-8001, USA \\ email: aklypin@nmsu.edu
}

\begin{abstract}
Current explanation of the overabundance of dark matter subhalos in the Local Group (LG) indicates that there maybe a limit on mass of a halo, which can host a galaxy. This idea can be tested using voids in the distribution of galaxies: at some level small voids should not contain any (even dwarf) galaxies. We use observational samples complete to $M_{B}=-12$ with distances less than $8 \mathrm{Mpc}$ to construct the void function (VF): the distribution of sizes of voids empty of any galaxies. There are $\sim 30$ voids with sizes ranging from 1 to $5 \mathrm{Mpc}$. We then study the distribution of dark matter halos in very high resolution simulations of the LCDM model. The theoretical VF matches the observations remarkably well only if we use halos with circular velocities larger than $45 \pm 10 \mathrm{~km} / \mathrm{s}$. This agrees with the Local Group predictions. There are smaller halos in the voids, but they should not produce any luminous matter. Small voids look quite similar to their giant cousins: the density has a minimum at the center of a void and it increases as we get closer to the border. Small nonluminous halos inside the void form a web of tiny filaments. Thus, both the Local Group data and the nearby voids indicate that isolated halos below $45 \pm 10 \mathrm{~km} / \mathrm{s}$ must not host galaxies and that small (few Mpc) voids are truly dark.
\end{abstract}

Keywords. galaxies: structure, statistics, halos; cosmology: large-scale structure of universe, dark matter

\section{Introduction}

The observational discovery of giant voids (Gregory \& Thompson (1978); Joeveer \& Einasto (1978); Kirshner et al. (1981)) was soon followed by the theoretical understanding that voids constitute a natural outcome of structure formation via gravitational instability (Peebles (1982); Hoffman \& Shaham (1982)). Emptiness of voids - the number of small galaxies in the voids - is an interesting question for both the observations and the theory to tackle: do we have a problem (Peebles (2001))? Cosmological simulations predict (e.g., Gottlöber et al. (2003)) that many small DM halos should reside in voids. There seems to be no disagreement between the LCDM theory and the observations (Patiri et al. (2006)) regarding the giant voids defined by $M_{*}$ galaxies or by $10^{12} M_{\odot}$ halos. The situation is less clear on smaller scales. In the region of $\sim 10 \mathrm{Mpc}$ around the Milky Way, where observations go to remarkably low luminosities, small voids look very empty: dwarf galaxies do not show a tendency to fill the voids and voids are still

$\dagger$ Present address: Konstantinova street, 4, app. 40, 188308, Gatchina, Leningradsky region, Russia. 
relatively large. The theory predicts that many dwarf dark matter halos should be in the voids, which puts it on a collision course with observations. Yet, below some mass the halos are expected to stop producing galaxies inside them. There are different arguments for that: stellar feedback (Dekel \& Silk (1986)) or photoionozation may play significant role in quenching star formation in too small halos. Still, it is difficult to get a definite answer because the physics of dwarfs at high redshifts is quite complicated.

Satellites of the Local Group give a more definite answer. Current explanation of the overabundance of the dark matter subhalos (Kravtsov et al. (2004)) assumes that dwarf halos above $V_{c} \approx 50 \mathrm{~km} / \mathrm{s}$ were forming stars before they fall into the Milky Way or M31. Once they fall in, they get severely stripped and may substantially reduce their circular velocity producing galaxies such as Draco or Fornax with the rms line-of-sight velocities only few $\mathrm{km} / \mathrm{s}$. The largest subhalos retain their gas and continue to form stars, while smaller ones may lose the gas and become dwarf spheroidals. Halos below the limit never had substantial star formation. They are truly dark. This scenario implies that $V_{c} \approx 50 \mathrm{~km} / \mathrm{s}$ is the limit for star formation in halos. If this picture is correct, it can be tested with small-size voids: they must be empty of any galaxies and are filled with gas and dark matter halos.

\section{DATA: Local Volume}

The first catalog of galaxies within 10 Mpc was compiled by Kraan-Korteweg \& Tammann (1979) and contained 179 galaxies. Tully (1988) noted that the Local Supercluster contains a number of filaments and that those outline the so-called Local Void, which begins just outside the Local Group and extends in the direction of the North Pole of the LSC. The Local Void looks practically free from galaxies. Over the past few years special searches for new nearby dwarf galaxies have been undertaken using numerous observational data: the optical sky survey POSS-II/ESO/SERC, HI, and on infrared surveys of the zone of avoidance, HIPASS and HIJASS. At present, the sample of galaxies with distances less than $10 \mathrm{Mpc}$ lists about 500 galaxies. For half of them the distances have been measured to an accuracy as high as 8-10\% (Karachentsev et al. (2004)). Over the last 5 years snapshot surveys with Hubble Space Telescope (HST) have provided us with the TRGB distances for many nearby galaxies. The absence of the "finger of God" effect in the Local Volume simplifies the analysis of the shape and orientation of nearby voids. Observations of the Local Volume have detected dwarf systems down to extremely low luminosity. This gives us a unique chance to detect voids which may be empty of any galaxies. Tikhonov \& Karachentsev (2006) analyzed nearby voids. Here we continue the analyse using an updated list of galaxies (Karachentsev, private communication). The volume limited sample is complete for galaxies with abs. magnitudes $M_{B}=-12$ within $8 \mathrm{Mpc}$ radius.

\section{Simulations}

We use N-body simulations done with the Adaptive Refinement Tree code (Kravtsov et al. (1997)). The simulations are for a spatially flat cosmological LCDM model with following parameters: $\Omega_{0}=0.7, \Omega_{\Lambda}=0.3 ; \sigma_{8}=0.9 ; H_{0}=70 \mathrm{~km} / \mathrm{s} / \mathrm{Mpc}$. As a measure of how large is a halo we typically use the maximum circular velocity $V_{c}=(G M / R)^{1 / 2}$. This quantity is easier to relate to observations as compared with the virial mass. For reference, halos with $V_{c}=50 \mathrm{~km} / \mathrm{s}$ have virial mass about $10^{10} M_{\odot}$ and halos with $V_{c}=20 \mathrm{~km} / \mathrm{s}$ have virial mass about $10^{9} M_{\odot}$. We use two simulations: (1) Box $80 \mathrm{Mpc} / \mathrm{h}$ (Box80); mass per particle $3 \times 10^{8} h^{-1} M_{\odot}$; simulations cover the whole volume and (2) 
Box $80 \mathrm{Mpc} / \mathrm{h}$ (Box $80 \mathrm{~S}$ ); spherical region of $10 \mathrm{Mpc}$ inside $80 \mathrm{Mpc} / \mathrm{h}$ box resolved with $5 \times 10^{6} h^{-1} M_{\odot}$ particles. We use halos resolved bay at least with 20 particles.

\section{Detecting Voids}

In order to detect voids, we place a $3 \mathrm{~d}$ mesh on the observational or simulation volume. We then find initial centers of voids as the mesh centers having the largest distances to nearest objects. In the next iteration, an initial spherical void may be increased by adding additional off-center empty spheres with smaller radius. The radius of the spheres is limited to be larger then 0.9 of the initial sphere and their centers must stay inside the volume of the first sphere. The process is repeated a few time. It produces voids which are slightly aspherical, but voids never become more flattened than 1:2 axial ratio. Artificial objects are placed on the boundaries of the sample to prevent voids getting out of the boundaries of the sample. We define the cumulative void function (CVF) as the fraction of the total volume occupied by voids with effective radius larger than $R_{\text {eff }}=\left(3 V_{\text {void }} / 4 \pi\right)^{-1 / 3}$.

\section{Cumulative Void Function of the Local Volume}

In order to construct CVF of the Local Volume we use two samples: (1) Galaxies brighter than $M_{B}=-12$ inside a sphere of radius $8 \mathrm{Mpc}$ and (2) all galaxies inside $7.5 \mathrm{Mpc}$. Results are present in the right panel of Figure 1. There are about 30 voids in the observational sample. We limit the radius of voids to be more than $1 \mathrm{Mpc}$. The two subsamples indicate some degree of stability: inclusion of a few low-luminosity galaxies does not change the void function.

We use the Box80 simulation (full volume) to construct a sample of 40 "Local Volumes". The selecton criteria are: 1) no halos with $M>10^{14} M_{\odot}$ inside a $8 \mathrm{Mpc}$ sphere (thus, no clusters in a sample); 2) the sphere must be centered on a halo with $150<$ $V_{c}<200 \mathrm{~km} / \mathrm{s}$ (Milky Way analog). Because of the mass resolution, the halo catalogs are complete down to halos with circular velocity $V_{c}=40 \mathrm{~km} / \mathrm{s}$. The second simulation (Box80S) provides one sample and it is complete down to $20 \mathrm{~km} / \mathrm{s}$.

The left panel in the Figure 1 shows CVF for different samples of halos and the observed CVF. Results indicate that voids in the distribution of halos with $V_{c}>45 \mathrm{~km} / \mathrm{s}$ give the best fit to the observed CVF. The theoretical CVF goes above the observational data if we use circular velocities larger than $60 \mathrm{~km} / \mathrm{s}$. If we use significantly lower limits, then the theory predicts too few large voids. The theoretical results match the observations if we use $V_{\text {circ }}=45 \pm 10 \mathrm{~km} / \mathrm{s}$. In this case, the match is remarkably good: the whole spectrum of voids is reproduced by the theory.

According to LCDM simulations totally empty front part of the Local Void is probable (this is not evident since Local (Tully) Void partly lies in the Zone of Avoidance). In a sample of ten $8 \mathrm{Mpc}$ "Local Volumes" a half of cases have voids comparable to the largest voids in LV if we consider entire LV sample.

\section{Interiors of voids in LCDM}

Density of matter inside voids increases toward borders of voids. This indicates that voids are physical; not statistical flukes because the same behaviour was found for giant voids in simulations of Gottlöber et al. (2003). To large degree the small and giant voids are similar. For example, there are very small filaments made of tiny halos inside small voids. Small voids can contain very few small halos. When studiing voids defined by 


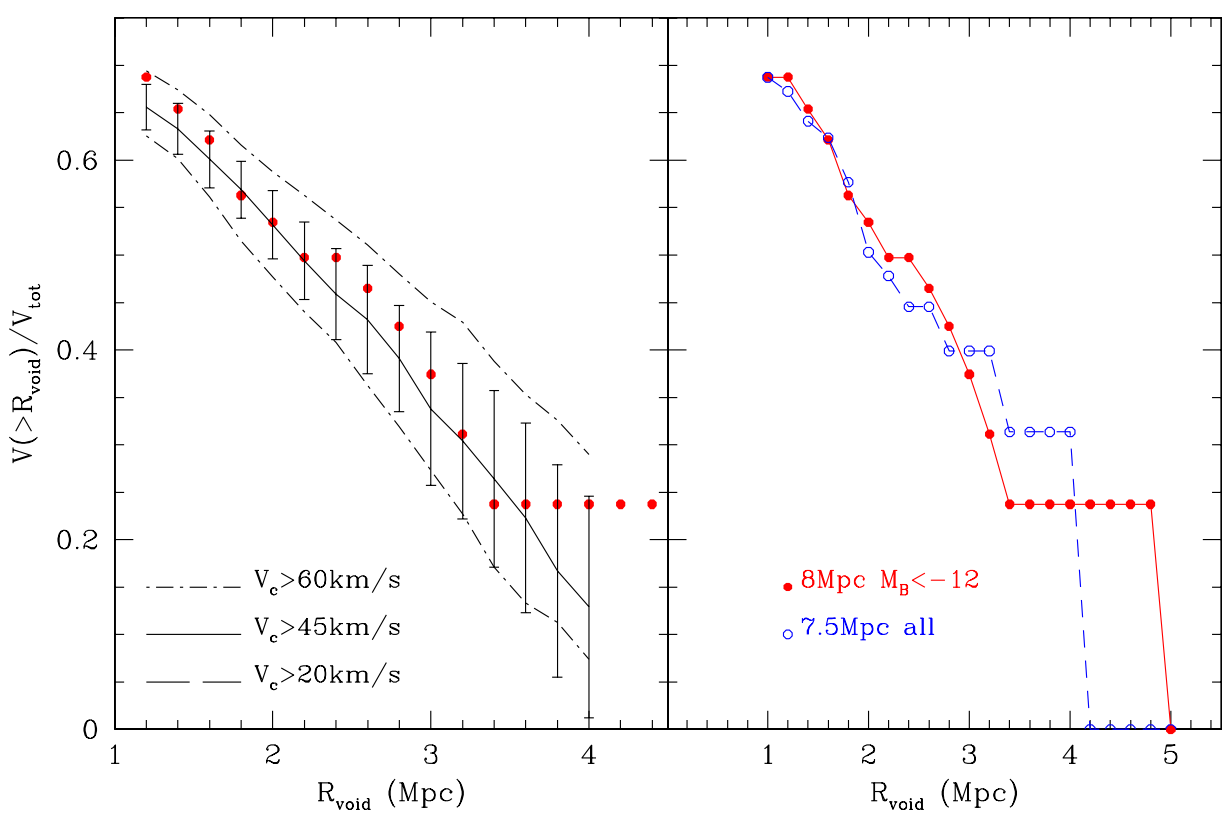

Figure 1. Right panel: the void function for two observational samples. The full curve and filled circles are for a complete volume limited sample with $M_{B}<-12$ and $R<8 \mathrm{Mpc}$. The open circles are for all oserved galaxies inside $7.5 \mathrm{Mpc}$. Comparison of the samples shows reasonable stability of the void function. Left panel: Observational data (the complete sample) are compared with the distribution of voids in samples of halos with different limits on halo circular velocity. CVF for $V_{c}=45 \mathrm{~km} / \mathrm{s}$ provides a remarkably good fit to observations. Note that the LCDM model predicts very large empty regions.

$V_{c}>45 \mathrm{~km} / \mathrm{s}$, we often find relatively large voids (few Mpc radius) totally empty of halos with $V_{c}>20 \mathrm{~km} / \mathrm{s}$.

\section{Luminosity funcion of LV}

The luminosity function of galaxies in the Local Volume, complete sample $M_{B}<-12$, is peaked on $M_{B} \sim-14$. The peak is mostly produced by isolated galaxies. Though it's statistical significance is still questionable, galaxies of this luminosity can be associated with DM halos with $V_{c} \sim 35-40 \mathrm{~km} / \mathrm{s}$. Taking into accout our main result - limit of appearance on $V_{c}=45 \pm 10 \mathrm{~km} / \mathrm{s}$ the bump in LF may be real. Better data are needed to make more definite conclusions. In particular, measurements of the rotation velocity function of LV galaxies is needed to find if there is something special about galaxies, which are close to the limit $V_{c}=30-50 \mathrm{~km} / \mathrm{s}$. They are isolated dwarf irregulars, which have large gas mass. Is it enough to slightly boost their luminocity and produce a maxium in the luminocity function before it dramatically decreases for smaller halos?

\section{Conclusions}

We find that

- The LCDM model is consistent with the cumulative volume functions of voids in the distribution of galaxies for a large luminosity range. According to LCDM, large empty voids in Local Volume such as the Local Void are probable. 
- There are significant (up to few Mpc) holes in the distribution of halos predicted by LCDM that are free from haloes with $V_{c}>20 \mathrm{~km} / \mathrm{s}$ : any haloes of astronomical interest.

- Voids in the distribution of haloes with $V_{c}>45 \pm 10 \mathrm{~km} / \mathrm{s}$ reproduce the Cumulative Void Function of the Local Volume galaxy sample. We can treat this value as a limit of appearance of a galaxy in a DM halo.

- $M_{B} \sim-14$ isolated galaxies (probably having $V_{c} \sim 35-45 \mathrm{~km} / \mathrm{s}$ ) may be on the limit of appearance of a galaxy in a DM halo. We speculate that the peak in Luminosity Function at this magnitude may be real. More data is needed to test this conclusion.

- Dark halos are probably located close to borders of voids.

\section{Acknowledgements}

We thank I.D. Karachentsev for providing us an updated list of his Catalog of Neighboring galaxies. A. Klypin acknowledges support of NSF grants to NMSU. Computer simulations used in this research were conducted on the Columbia supercomputer at the NASA Advanced Supercomputing Division and at the Leibniz-Rechenzentrum (LRZ), Munchen, Germany. A. Tikhonov acknowledges support of grant no. MK-6899.2006.2 from the President of Russia.

\section{References}

Dekel, A., \& Silk, J. 1986, ApJ, 303, 39

Gottlöber, S., Łokas, E. L., Klypin, A., \& Hoffman, Y. 2003, MNRAS, 344, 715

Gregory, S. A., \& Thompson, L. A. 1978, ApJ, 222, 784

Hoffman, Y., \& Shaham, J. 1982, ApJ Lett., 262, L23

Peebles, P. J. E. 1982, ApJ, 257, 438

Joeveer, M., \& Einasto, J. 1978, Large Scale Structures in the Universe, 79, 241

Kirshner, R. P., Oemler, A., Jr., Schechter, P. L., \& Shectman, S. A. 1981, ApJ Lett., 248, L57

Karachentsev, I. D., Karachentseva, V. E., Huchtmeier, W. K., \& Makarov, D. I. 2004, AJ, 127, 2031

Kraan-Korteweg, R. C., \& Tammann, G. A. 1979, Astronomische Nachrichten, 300, 181

Kravtsov, A. V., Klypin, A. A., \& Khokhlov, A. M. 1997, ApJ Suppl., 111, 73

Kravtsov, A. V., Gnedin, O. Y., \& Klypin, A. A. 2004, ApJ, 609, 482

Patiri, S. G., Prada, F., Holtzman, J., Klypin, A., \& Betancort-Rijo, J. 2006, MNRAS, 372, 1710

Peebles, P. J. E. 2001, ApJ, 557, 495

Tikhonov, A. V., \& Karachentsev, I. D. 2006, ApJ, 653, 969

Tully, R. B., \& Fisher, J. R. 1987, Atlas of Nearby Galaxies, Cambridge University Press. 